\title{
A Survey of the Implementation of Numerical Schemes for the Heat Equation Using Forward Euler in Time
}

\author{
Pedro Pablo Cárdenas Alzate \\ Department of Mathematics, Universidad Tecnológica de Pereira, Pereira, Colombia \\ Email: ppablo@utp.edu.co \\ Received 16 September 2014; revised 17 October 2014; accepted 22 October 2014 \\ Copyright (C) 2014 by author and Scientific Research Publishing Inc. \\ This work is licensed under the Creative Commons Attribution International License (CC BY). \\ http://creativecommons.org/licenses/by/4.0/ \\ c) (i) Open Access
}

\begin{abstract}
We establish the conditions for the compute of the Global Truncation Error (GTE), stability restriction on the time step and we prove the consistency using forward Euler in time and a fourth order discretization in space for Heat Equation with smooth initial conditions and Dirichlet boundary conditions.
\end{abstract}

\section{Keywords}

\section{Global Truncation, Forward Euler, Heat Equation}

\section{Introduction}

In this paper we have considered the heat equation $u_{t}=C u_{x x}$ on $[0,1] \times[0, T]$ with smooth initial conditions and Dirichlet boundary conditions $\left(C \in \mathbb{R}^{+}\right)$. Using forward Euler in time and fourth order discretization in space, we compute the Global Truncation Error (GTE), the stability restriction on the time step $\Delta t$, also we prove consistency and finally we prove the convergence for this scheme.

Much attention has been paid to the development, analysis and implementation of accurate methods for the numerical solution of this problem in the literature. Many problems are modeled by smooth initial conditions and Dirichlet boundary conditions. A number of procedures have been suggested (see, for instance [1]-[3]). We can say that three classes of solution techniques have emerged for solution of PDE: the finite difference techniques, the finite element methods and the spectral techniques. The last one has the advantage of high accuracy attained by the resulting discretization for a given number of nodes [4]-[7]. Let $\Delta x$ denote the grid-size in the spatial direction and $\Delta t$ the gridsize in the time direction. By using forward Euler in time, and the fourth order 
discretization from the previous problem in space, the heat equation reads:

$$
\frac{u_{i}^{n+1}-u_{i}^{n}}{\Delta t}=C \frac{-u_{i-2}^{n}+16 u_{i-1}^{n}-30 u_{i}^{n}+16 u_{i+1}^{n}-u_{i+2}^{n}}{12 \Delta x^{2}}
$$

We'll assume that the discretizations used near the boundaries have the same order [8] and [9].

\section{Global Truncation Error (GTE)}

There are three equivalent ways of computing the Global Truncation Error for this case.

Way 1. We can always go back to the definition of the GTE. Let $U^{n}$ be the true solution at stage $n$, and $v^{n}$ be the solution returned by the scheme at stage $n$. Therefore

$$
E^{n}=v^{n}-U^{n}
$$

We consider de LTE

$$
\begin{gathered}
\tau=\frac{u(x, t+\Delta t)-u(x, t)}{\Delta t} \\
-\frac{C}{12(\Delta x)^{2}}[-u(x-2 \Delta x, t)+16 u(x-\Delta x, t)-30 u(x, t)+16 u(x+\Delta x, t)-u(x+2 \Delta x, t)] \\
\tau_{i}^{n}=\frac{u_{i}^{n+1}-u_{i}^{n}}{\Delta t}-\frac{C}{12(\Delta x)^{2}}\left[-u_{i-2}^{n}+16 u_{i-1}^{n}-30 u_{i}^{n}+16 u_{i+1}^{n}-u_{i+2}^{n}\right] \\
u_{i}^{n+1}=u_{i}^{n}+\frac{C \Delta t}{12(\Delta x)^{2}}\left[u_{i-2}^{n}+16 u_{i-1}^{n}-30 u_{i}^{n}+16 u_{i+1}^{n}-u_{i+2}^{n}\right]+\Delta t \tau_{i}^{n} \\
u_{i}^{n+1}=\frac{C \Delta t}{12(\Delta x)^{2}}\left[u_{i-2}^{n}+16 u_{i-1}^{n}+\left(\frac{12(\Delta x)^{2}}{C \Delta t}-30\right) u_{i}^{n}+16 u_{i+1}^{n}-u_{i+2}^{n}\right]+\Delta t \tau_{i}^{n} \\
u_{i}^{n+1}=\frac{C \Delta t}{12(\Delta x)^{2}}\left(-1,16, \frac{12(\Delta x)^{2}}{C \Delta t}-30,16,-1\right)\left(\begin{array}{l}
u_{i-2}^{n} \\
u_{i-1}^{n} \\
u_{i}^{n} \\
u_{i+1}^{n} \\
u_{i+2}^{n}
\end{array}\right)+\Delta t \tau_{i}^{n}
\end{gathered}
$$

So that at stage $n$, we have

$$
U^{n+1}=\frac{C \Delta t}{12(\Delta x)^{2}} B(\Delta t, \Delta x) U^{n}+\Delta t \tau^{n}+\beta^{n}
$$

where

$$
U^{n}=\left(\begin{array}{c}
u_{0}^{n} \\
u_{1}^{n} \\
\vdots \\
u_{M-1}^{n} \\
u_{M}^{n}
\end{array}\right) \quad \text { and } \quad \tau^{n}=\left(\begin{array}{c}
\tau_{0}^{n} \\
\tau_{1}^{n} \\
\vdots \\
\tau_{M-1}^{n} \\
\tau_{M}^{n}
\end{array}\right)
$$

$\beta^{n}$ is a vector taking care of the boundary conditions and $B(\Delta t, \Delta x)$ is a matrix. Since

$$
v^{n+1}=\frac{C \Delta t}{12(\Delta x)^{2}} B(\Delta t, \Delta x) v^{n}+\beta^{n}
$$


we get at stage $N$

$$
\begin{gathered}
E^{N}=v^{N}-U^{N}=\frac{C \Delta t}{12(\Delta x)^{2}} B(\Delta t, \Delta x)\left(v^{N-1}-U^{N-1}\right)-\Delta t \tau^{N} \\
=\frac{C \Delta t}{12(\Delta x)^{2}} B(\Delta t, \Delta x)\left(\frac{C \Delta t}{12(\Delta x)^{2}} B(\Delta t, \Delta x)\left(v^{N-2}-U^{N-2}\right)-\Delta t \tau^{N-2}\right)-\Delta t \tau^{N-1} \\
=\left(\frac{C \Delta t}{12(\Delta x)^{2}}\right)^{2} B^{2}(\Delta t, \Delta x)\left(v^{N-2}-U^{N-2}\right)-\Delta t\left(\frac{C \Delta t}{12(\Delta x)^{2}} B(\Delta t, \Delta x) \tau^{N-2}-\tau^{N-1}\right) \\
=\left(\frac{C \Delta t}{12(\Delta x)^{2}}\right)^{N} B^{N}(\Delta t, \Delta x)\left(v^{0}-U^{0}\right)-\Delta t \sum_{n=1}^{N}\left(\frac{C \Delta t}{12(\Delta x)^{2}}\right)^{N-n} B^{N-n}(\Delta t, \Delta x) \tau^{N-n} \\
=\left(\frac{C \Delta t}{12(\Delta x)^{2}}\right)^{N} B^{N}(\Delta t, \Delta x) E^{0}-\Delta t \sum_{n=1}^{N}\left(\frac{C \Delta t}{12(\Delta x)^{2}}\right)^{N-n} B^{N-n}(\Delta t, \Delta x) \tau^{N-n}
\end{gathered}
$$

We now wish to estimate this quantity: first using the triangle inequality, we get

$$
\mathrm{GTE}=\left\|E^{N}\right\| \leq\left\|\left(\frac{C \Delta t}{12(\Delta x)^{2}}\right)^{N} B^{N}(\Delta t, \Delta x) E^{0}\right\|+\Delta t\left\|\sum_{n=1}^{N}\left(\frac{C \Delta t}{12(\Delta x)^{2}}\right)^{N-n} B^{N-n}(\Delta t, \Delta x) \tau^{N-n}\right\|
$$

Now, taking stability into account, we can see that $\left\|B\left((\Delta x)^{2}, \Delta x\right)\right\|=O(1)$. Letting $T=N \Delta t$ we get

$$
\begin{gathered}
\left\|E^{N}\right\| \leq\left\|\left(\frac{C \Delta t}{12(\Delta x)^{2}}\right)^{N} B^{N}(\Delta t, \Delta x) E^{0}\right\|+\Delta t\left\|\sum_{n=1}^{N}\left(\frac{C \Delta t}{12(\Delta x)^{2}}\right)^{N-n} B^{N-n}(\Delta t, \Delta x) \tau^{N-n}\right\| \\
=\left\|O(1) \Delta O(1) E^{0}\right\|+\Delta t\left\|\sum_{n=1}^{N} O(1) \Delta O(1) \tau^{N-n}\right\| \\
\leq\left\|E^{0}\right\|+\Delta t \sum_{n=1}^{N}\left\|\tau^{N-n}\right\| \leq\left\|E^{0}\right\|+\Delta t N\left\|\tau^{N-n}\right\| \leq\left\|E^{0}\right\|+T\left\|\tau^{N-n}\right\|
\end{gathered}
$$

Now, assuming that initial error is not too large, we have

$$
\operatorname{GTE} \leq\left\|\tau^{N-n}(\Delta t, \Delta x)\right\| \underset{\text { (by stability })}{=}\left\|\tau^{N-n}\left((\Delta x)^{2}, \Delta x\right)\right\|=O\left((\Delta x)^{2}+(\Delta x)^{4}\right)=O\left((\Delta x)^{2}\right)
$$

Finally, we can conclude that the GTE $\propto O\left((\Delta x)^{2}\right)$

Way 2. The GTE can be estimated by computing the LTE $\tau_{i}^{n}(\Delta t, \Delta x)$ and imposing stability to it

$$
\mathrm{GTE} \leq \tau_{i}^{n}(\Delta t, \Delta x) \underset{\text { (by stability) }}{=} \tau_{i}^{n}\left((\Delta x)^{2}, \Delta x\right)=O\left((\Delta x)^{2}+(\Delta x)^{4}\right)=O\left((\Delta x)^{2}\right)
$$

Way 3. We can also compute the one-step-error for the scheme. This quantity is basically equal to $\Delta t \cdot \tau_{i}^{n}$ since it is computed as follows

$$
\frac{u_{i}^{n+1}-u_{i}^{n}}{\Delta t}=C \frac{-u_{i-2}^{n}+16 u_{i-1}^{n}-30 u_{i}^{n}+16 u_{i+1}^{n}-u_{i+2}^{n}}{12(\Delta x)^{2}}
$$




$$
\Leftrightarrow u_{i}^{n+1}=u_{i}^{n}+C(\Delta t) \frac{-u_{i-2}^{n}+16 u_{i-1}^{n}-30 u_{i}^{n}+16 u_{i+1}^{n}-u_{i+2}^{n}}{12(\Delta x)^{2}}
$$

then substitute the true solution and compute the difference of the two sides

$$
\begin{aligned}
\alpha_{i}^{n} & =u(x, t)+C(\Delta t)\left(\frac{-u(x-2 \Delta x)+16 u(x-\Delta x)-30 u(x)+16 u(x+\Delta x)-u(x+2 \Delta x)}{12(\Delta x)^{2}}\right)-u(x, t+\Delta t) \\
& =O\left(\Delta t(\Delta x)^{4}+(\Delta x)^{2}\right) .
\end{aligned}
$$

We can then estimate the GTE by summing up the one-step error at each stage

$$
\begin{gathered}
\text { GTE } \leq\left\|\sum_{n=1}^{N} \alpha^{n}\right\| \leq N\left\|\alpha^{n}\right\|=\frac{T}{\Delta t} O\left(\Delta t(\Delta x)^{4}+(\Delta x)^{2}\right) \\
\underset{\text { (by stability) }}{=} \frac{T}{(\Delta x)^{2}} O\left((\Delta x)^{2}(\Delta x)^{4}+(\Delta x)^{2}\right)=O\left((\Delta x)^{2}\right)
\end{gathered}
$$

\section{Stability Restriction}

We start by computing the stability restriction one has to impose on $\Delta t$. We apply Von Neumann stability analysis to the scheme: Letting $k$ denote the wave number, we get

$$
\begin{aligned}
\frac{G-1}{\Delta t} & =C \frac{-\mathrm{e}^{-2 i k \Delta x}+16 \mathrm{e}^{-i k \Delta x}-30+16 \mathrm{e}^{i k \Delta x}-\mathrm{e}^{2 i k \Delta x}}{12(\Delta x)^{2}} \\
& =\frac{C}{3(\Delta x)^{2}} \frac{1}{4}\left(-\mathrm{e}^{-2 i k \Delta x}+16 \mathrm{e}^{-i k \Delta x}-30+16 \mathrm{e}^{i k \Delta x}-\mathrm{e}^{2 i k \Delta x}\right) \\
& =-\frac{C}{3(\Delta x)^{2}} \frac{1}{4}\left(\mathrm{e}^{-2 i k \Delta x}-16 \mathrm{e}^{-i k \Delta x}+30-16 \mathrm{e}^{i k \Delta x}+\mathrm{e}^{2 i k \Delta x}\right) \\
& =-\frac{C}{3(\Delta x)^{2}} \frac{1}{4}\left(28+\mathrm{e}^{2 i k \Delta x}+2+\mathrm{e}^{-2 i k \Delta x}-16 \mathrm{e}^{-i k \Delta x}-16 \mathrm{e}^{i k \Delta x}\right) \\
& =-\frac{C}{3(\Delta x)^{2}} \frac{1}{4}\left(7+\frac{\mathrm{e}^{2 i k \Delta x}+2+\mathrm{e}^{-2 i k \Delta x}}{4}-8 \frac{\mathrm{e}^{i k \Delta x}+\mathrm{e}^{-i k \Delta x}}{2}\right) \\
& =-\frac{C}{3(\Delta x)^{2}}\left(7+\cos ^{2}(k \Delta x)-8 \cos (k \Delta x)\right)
\end{aligned}
$$

then

$$
G(k)=1-\frac{C \Delta t}{3(\Delta x)^{2}}\left(\cos ^{2}(k \Delta x)-8 \cos (k \Delta x)+7\right)
$$

Now, let $y=\cos (k \Delta x)$ and

$$
w(y)=y^{2}-8 y+7=(y-7)(y-1)
$$

So that $w \geq 0$ when $y=\cos (k \Delta x) \in[-1,1]$. This guarantees that $G(k)<1$. Now, in order to make sure that $|G(k)| \leq 1$, we must have

$$
-1 \leq G(k) \Leftrightarrow-1 \leq 1-\frac{C \Delta t}{3(\Delta x)^{2}}\left(\cos ^{2}(k \Delta x)-8 \cos (k \Delta x)+7\right)
$$




$$
\begin{gathered}
\Leftrightarrow \frac{C \Delta t}{3(\Delta x)^{2}}\left(\cos ^{2}(k \Delta x)-8 \cos (k \Delta x)+7\right) \leq 2 \\
\Leftrightarrow \frac{C \Delta t}{3(\Delta x)^{2}} \max \left(\cos ^{2}(k \Delta x)-8 \cos (k \Delta x)+7\right) \leq 2 \\
\Leftrightarrow \frac{C \Delta t}{3(\Delta x)^{2}}\left((-1)^{2}-8(-1)+7\right) \leq 2 \\
\Leftrightarrow \frac{16 C \Delta t}{3(\Delta x)^{2}} \leq 2 \\
\Leftrightarrow \Delta t \leq \frac{3}{8} \frac{(\Delta x)^{2}}{C} \quad(\text { stability criterion) }
\end{gathered}
$$

\section{Consistency and Convergence}

We know that a discretization scheme [10] for a PDE is consistent provided that $\tau(\Delta x, \Delta t) \rightarrow 0$ as $\Delta x, \Delta t \rightarrow 0$, where $\tau$ is the LTE. We compute it by substituting the true solution in the scheme and by using Taylor expansions

$$
\begin{aligned}
& \tau(\Delta x, \Delta t)= \frac{u(x, t+\Delta t)-u(x, t)}{\Delta t} \\
&-C \frac{-u(x-2 \Delta x, t)+16 u(x-\Delta x, t)-30 u(x, t)+16 u(x+\Delta x, t)-u(x+2 \Delta x, t)}{12(\Delta x)^{2}} \\
&= u_{t}+\frac{\Delta t}{2} u_{t t}+\cdots-C\left(u_{x x}+O\left((\Delta x)^{4}\right)\right) \\
&=\underbrace{u_{t}-C u_{x x}}_{=0}+O\left(\Delta t+(\Delta x)^{4}\right)=O\left(\Delta t+(\Delta x)^{4}\right)
\end{aligned}
$$

Thus, $\tau$ obviously goes to 0 as $\Delta x$ and $\Delta t$ go to 0 . Therefore, we can say that the scheme is consistent.

Lastly, since we proved that the scheme is consistent and stable, by Lax equivalence theorem, we prove that the scheme is convergent. (By the above, since the GTE is $O\left((\Delta x)^{2}\right)$, it goes to 0 as $\Delta x \rightarrow 0$ ). We can see that Lax Equivalence Theorem for PDEs holds provided the scheme is linear (which is the case here). It may not hold for non-linear schemes.

Another way to get the one-step error for the scheme is to combine the LTE for the temporal and spatial discretization, as follows.

LTE for forward Euler is $O\left((\Delta t)^{2}\right)$ and the LTE for the spatial discretization is

$$
\begin{aligned}
\tau(\Delta x) & =-u(x-2 \Delta x)+16 u(x-\Delta x)-30 u(x)+16 u(x+\Delta x)-u(x+2 \Delta x)-1(\Delta x)^{2} u_{x x} \\
& =12(\Delta x)^{2}\left(\frac{-u(x-2 \Delta x)+16 u(x-\Delta x)-30 u(x)+16 u(v)-u(x+2 \Delta x)}{12(\Delta x)^{2}}-u_{x x}\right) \\
& =12(\Delta x)^{2} O\left((\Delta x)^{4}\right)=O\left((\Delta x)^{6}\right)
\end{aligned}
$$

This is equivalent to the previous method for getting the one-step error.

\section{Acknowledgements}

I would like to thank the referee for his valuable suggestions that improved the presentation of this paper and my 
gratitude to Department of Mathematics of the Universidad Tecnológica de Pereira (Colombia) and the group GEDNOL.

\section{References}

[1] Strikwerda, J.C. (1989) Finite Difference Schemes and Partial Differential Equations. Wadsworth \& Brooks, USA.

[2] McRea, G.J. and Godin, W.R. (1967) Numerical Solution of Atmospheric Diffusion for Chemically Reacting Flows. Journal of Computational Physics, 77, 1-42.

[3] Hundsdorfer, W. and Koren, B. (1995) A Positive Finite-Difference Advection Scheme Applied on Locally Refined Grids. Journal of Computational Physics, 117, 35-36. http://dx.doi.org/10.1006/jcph.1995.1042

[4] Morton, K.W. (1980) Stability of Finite Difference Approximations to a Diffusion-Convection Equation. International Journal for Numerical Methods Engineering, 15, 677-683. http://dx.doi.org/10.1002/nme.1620150505

[5] Canuto, C. and Hussaini, M. (1988) Spectral Methods in Fluids Dynamics. Springer Series in Computational Physics, Springer-Verlag, Berlin. http://dx.doi.org/10.1007/978-3-642-84108-8

[6] Dehghan, M. (2005) On the Numerical Solution of the One-Dimensional Convection-Diffusion Equation. Mathematical Problems in Engineering, 1, 61-74.

[7] Dehghan, M. (2007) The One-Dimensional Heat Equation Subject to a Boundary Integral Specification. Chaos, Solitons \& Fractals, 32, 661-675. http://dx.doi.org/10.1155/MPE.2005.61

[8] Mitchell, A.R. and Griffiths, D.F. (1980) The Finite Difference Method in Partial Differential Equations. John Wiley \& Sons, Chichester.

[9] Mickens, R.E. (2000) Applications of Nonstandard Finite Differences Schemes. World Scientific Publishing, River Edge.

[10] Lu, X., et al. (2005) A New Analytical Method to Solve the Heat Equation for a Multi-Dimensional Composite Slab. Journal of Physics, 38, 2873. http://doi:10.1088/0305-4470/38/13/004 
Scientific Research Publishing (SCIRP) is one of the largest Open Access journal publishers. It is currently publishing more than 200 open access, online, peer-reviewed journals covering a wide range of academic disciplines. SCIRP serves the worldwide academic communities and contributes to the progress and application of science with its publication.

Other selected journals from SCIRP are listed as below. Submit your manuscript to us via either submit@scirp.org or Online Submission Portal.
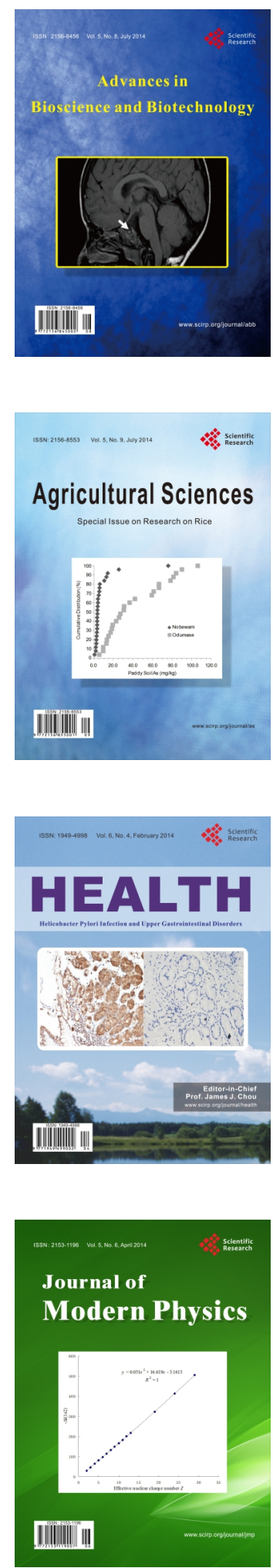
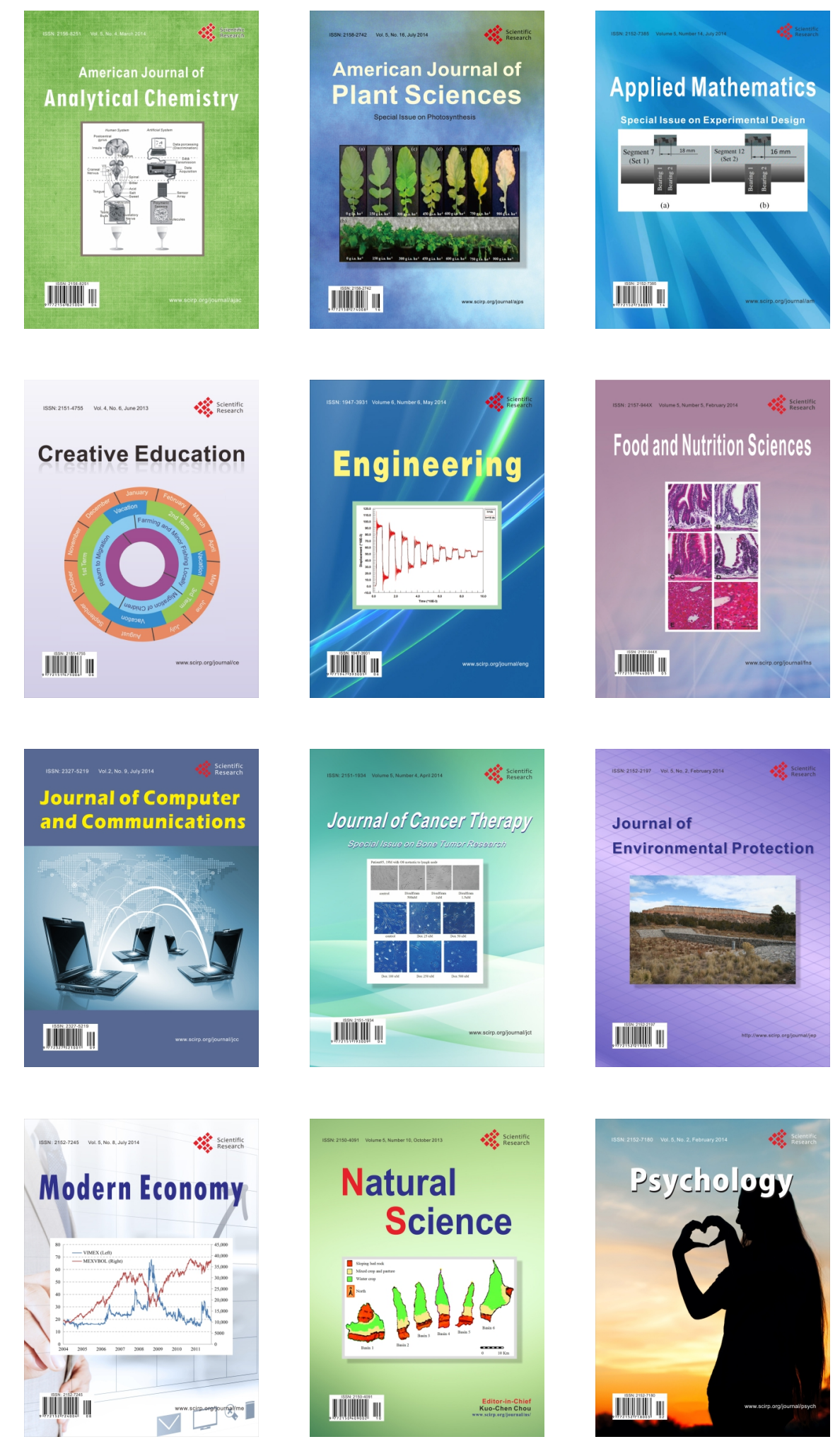